\title{
An Effective Scheduling-Based RFID Reader Collision Avoidance Model and Its Resource Allocation via Artificial Immune Network
}

\author{
Shanjin Wang, ${ }^{1}$ Zhonghua $\mathrm{Li}^{2}$ Chunhui $\mathrm{He}^{2}$ and Jianming $\mathrm{Li}^{2}$ \\ ${ }^{1}$ School of Electronic Engineering, Dongguan University of Technology, Dongguan, Guangdong 523808, China \\ ${ }^{2}$ School of Data and Computer Science, Sun Yat-Sen University, Guangzhou, Guangdong 510006, China
}

Correspondence should be addressed to Zhonghua Li; lizhongh@mail.sysu.edu.cn

Received 21 July 2015; Accepted 20 December 2015

Academic Editor: Nazrul Islam

Copyright (C) 2016 Shanjin Wang et al. This is an open access article distributed under the Creative Commons Attribution License, which permits unrestricted use, distribution, and reproduction in any medium, provided the original work is properly cited.

\begin{abstract}
Radio frequency identification, that is, RFID, is one of important technologies in Internet of Things. Reader collision does impair the tag identification efficiency of an RFID system. Many developed methods, for example, the scheduling-based series, that are used to avoid RFID reader collision, have been developed. For scheduling-based methods, communication resources, that is, time slots, channels, and power, are optimally assigned to readers. In this case, reader collision avoidance is equivalent to an optimization problem related to resource allocation. However, the existing methods neglect the overlap between the interrogation regions of readers, which reduces the tag identification rate (TIR). To resolve this shortage, this paper attempts to build a reader-to-reader collision avoidance model considering the interrogation region overlaps (R2RCAM-IRO). In addition, an artificial immune network for resource allocation (RA-IRO-aiNet) is designed to optimize the proposed model. For comparison, some comparative numerical simulations are arranged. The simulation results show that the proposed R2RCAM-IRO is an effective model where TIR is improved significantly. And especially in the application of reader-to-reader collision avoidance, the proposed RA-IRO-aiNet outperforms GA, opt-aiNet, and PSO in the total coverage area of readers.
\end{abstract}

\section{Introduction}

As a wireless automatic identification and data capture technique, RFID (radio frequency identification) is employed to automatically identify the targets. With the main advantages of noncontact and fast reading rate for multiple targets, RFID has been applied widely in logistics, supply chain management, transportation, manufacturing, positioning, and many other fields $[1,2]$.

For RFID applications with many tags within a large deployment region, multiple or even dense readers are necessary to enhance the identification efficiency. If R2RCP (reader-to-reader collision problem) happens, the identification efficiency of the systems will be reduced inevitably. Therefore, how to solve R2RCP has become one research focus. In general, scheduling-based reader collision avoidance methods are suitable for stationary reader scenarios. In the scheduling-based series, one or more kinds of communication resources, that is, time slots, channels, and power, are allocated to readers to maximize the tag identification efficiency of RFID systems. Further, intelligent algorithms are applied to optimize the utility of RFID systems. In [3], the reader collision problem is formulated as 0/1 integer programming problem and a simulated annealing algorithm is introduced to solve it. In [4], time slots are allocated by using color graphing and PSO. A resource allocation-based model is built in [5] and is optimized by using the genetic algorithm (RA-GA). Such model is improved as a readerto-reader collision avoidance model (R2RCAM) by [6]. And a well designed artificial immune system is introduced to optimize R2RCAM, which has better performance in solving R2RCP by comparison with RA-GA and a random method (RM). These achievements indicate that intelligent algorithms are effective to deal with the reader collision problems from the viewpoint of resource scheduling. Note that a family of coverage-based methods $[7,8]$ deal with the reader collision problem by adaptively controlling the communication power of readers and minimizing the overlap 
between the interrogation region. Inspired by such spirit, it is worth to consider the overlap of the interrogation regions of readers and the resource of power, while R2RCAM without consideration about overlap is not suitable for the practice of RFID applications.

In order to overcome the defects of canonical R2RCAM, this paper proposes an effective R2RCAM considering the interrogation region overlap (R2RCAM-IRO). The proposed R2RCAM-IRO takes into account not only the overlap but also the resource of power and the tag identification capacity of a reader. In addition, a revised artificial immune network (aiNet), namely, RA-IRO-aiNet, is designed to optimize R2RCAM-IRO. The proposed R2RCAM-IRO is expected to improve the tag identification rate with limited communication resources for multiple readers, where the proposed RAIRO-aiNet is used.

The rest of our paper is arranged as follows. Section 2 reviews related theories and principles including R2RCP, R2RCAM, and the basic artificial immune network. Section 3 proposes R2RCAM-IRO and RA-IRO-aiNet. And Section 4 proves the effectiveness of the proposed model and compares RA-IRO-aiNet with RA-GA [5], PSO [4], and opt-aiNet [9] in the area of effective interrogation region by numerical simulations. And finally, the conclusions are made in Section 5.

\section{Reviews of Related Theories and Principles}

2.1. Reader-to-Reader Collision Problem (R2RCP). A basic RFID system is composed of some tags, one or more readers, and an application system, as shown in Figure 1. Multiple readers operating within a group become a reader network. When a tag attached to the objects to be identified enters the interrogation region of a reader, it will be powered and activated. Then, the tag sends its corresponding information to the reader. The reader identifies the tag by receiving information and sends the corresponding data to related application system for further processing and storage. And the readers operate according to the commands of the application system.

In multiple-reader scenarios, the backscatter signal of a tag is easily disturbed. This is because the backscatter power is much weaker than the transmitting power of any neighboring interfering reader. As a result, R2RCP will happen and decrease the desired reader's effective interrogation radius.

Figure 2 shows an example of R2RCP with two readers, where one is a desired reader $R_{i}$ and the other is an interfering reader $R_{j}$. The distance between $R_{i}$ and $R_{j}$ is defined as $d_{i, j}$. If only $R_{i}$ is operating, that is, $R_{i}$ is not interfered; it can harvest a maximum interrogation radius with the value of $r_{\max }$. However, if $R_{j}$ is operating simultaneously with $R_{i}$, the interrogation radius of reader $R_{i}$ will reduce to $r$. Further, strong enough interference from $R_{j}$ will probably make $r$ smaller than $x$ which is the distance from the target tag $T$ to
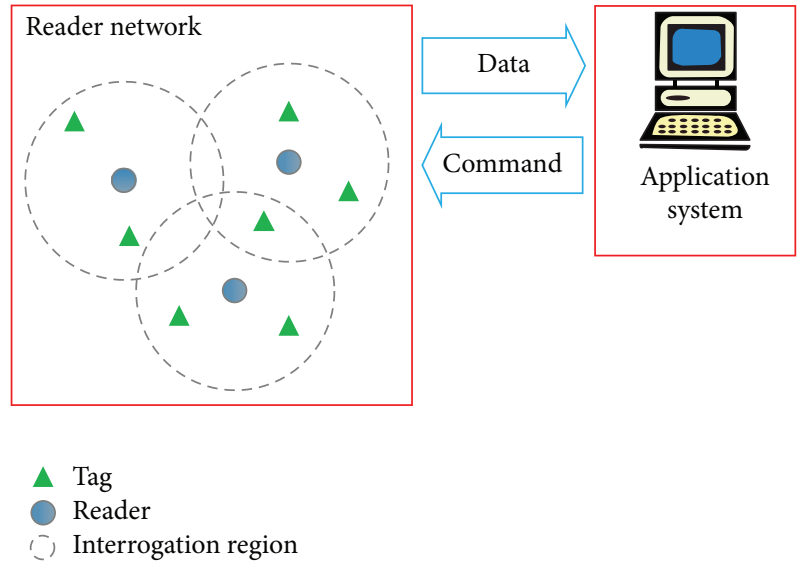

FIgURE 1: Schematic description of a basic RFID system.

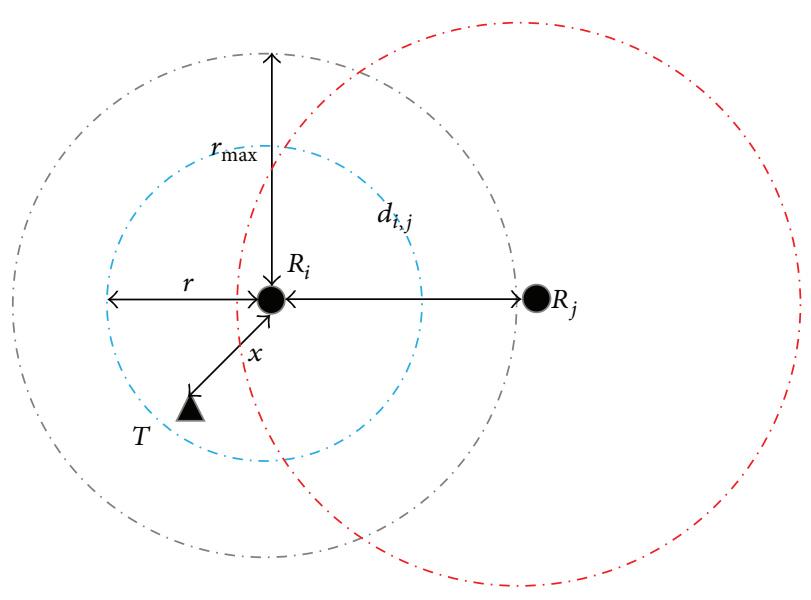

$R_{i}$ : desired reader

$R_{j}$ : interfering reader

$T$ : target tag

FIGURE 2: Reader-to-reader collision problem.

$R_{i}$. Consequently, $R_{i}$ is not able to detect $T$ successfully. To sum up, the radius $r_{i}$ of the desired reader $R_{i}$ follows

$r_{i}$

$= \begin{cases}r<r_{\max }, & \text { if } R_{i} \text { and } R_{j} \text { operates at the same time } \\ r_{\max }, & \text { otherwise. }\end{cases}$

2.2. Canonical Reader-to-Reader Collision Avoidance Model (R2RCAM). Assume that $N_{\text {rd }}$ readers are deployed randomly in a specified region with the area of $S_{0} . N_{\mathrm{Ch}}$ channels and $N_{S}$ slots are available to be allocated for these readers.

For clarification, three variables are defined as in Table 1. And $r_{i, \text { th }}^{k}$ can be calculated by solving

$$
r_{i, \text { th }}^{k}=\underset{x}{\arg \max } \operatorname{SINR}_{i}^{k}(x) \geq \operatorname{SINR}_{\text {min }}=\left(\frac{\alpha_{b \omega} E_{\mathrm{tg}} P_{i}^{k} G_{T} G_{R} \mathrm{PL}_{0}^{2}}{\operatorname{SINR}_{\min }\left(h G_{T} G_{R} \sum_{j=1, j \neq i}^{N_{\mathrm{rd}}} \omega_{j}^{k} P_{j}^{k} \beta_{m}\left(\left|\mathrm{CH}_{i}^{k}-\mathrm{CH}_{j}^{k}\right|\right) \mathrm{PL}_{0} d_{j, i}^{-\gamma}+N s_{i}\right)}\right)^{1 / 2 \gamma},
$$


TABLE 1: Definitions of the variables.

\begin{tabular}{ll}
\hline Var. & Definition \\
\hline$r_{i}^{k}$ & The actual interrogation radius of $R_{i}$ in $k$ th slot \\
$r_{i, \mathrm{th}}^{k}$ & The theoretical effective interrogation radius of $R_{i}$ in \\
& $k$ th slot \\
$r_{i, \max }^{k}$ & The theoretical maximum interrogation radius of $R_{i}$ \\
\hline
\end{tabular}

where $\operatorname{SINR}_{i}^{k}$ is the signal-to-interference-plus-noise ratio (SINR) for $R_{i}$ in the $k$ th slot, SINR $_{\text {min }}$ is the desired minimum value of SINR to identify tags, $\alpha_{b \omega}$ presents the normalized spectrum power, $E_{\mathrm{tg}}$ presents reflection coefficient of effective power, $G_{T}$ represents the gain of transmitting antenna, $G_{R}$ represents the gain of receiving antenna, $\mathrm{PL}_{0}$ is the referenced path loss at $1 \mathrm{~m}$ distant from a reader, $\gamma$ is the exponent of path loss that is not less than two, and $h$ denotes the fading coefficient. The signal fading is ignored due to the line-ofsight distance between readers. In addition, $P_{i}^{k}$ represents the operating power of $R_{i}$ in the $k$ th slot. According to EPC global C1G2 [10], $\beta_{m}$ denotes the spectrum mask level and is also a function with the arguments of the channel interval between readers. $\mathrm{CH}_{i}^{k}$ is the channel number used by $R_{i}$ in the $k$ th slot, where $\mathrm{CH}_{i}^{k}=1,2, \ldots, N_{S}$. Note that $\omega_{j}^{k}$ is a 2 -value function which equals 1 if $R_{j}$ is operating at the $k$ th slot and equals 0 otherwise. Please see [6] for detailed derivations.

However, $r_{i}^{k}$ must be not more than $r_{i \text {, max }}^{k}$; that is,

$$
r_{i}^{k} \leq r_{i, \max }^{k}=\left(\frac{\lambda_{i}^{k}}{4 \pi}\right) \sqrt{\frac{P_{i}^{k} G_{T} G_{R}}{P_{\min }} \cdot \frac{1-M_{d}^{4}}{\left(1+M_{d}\right)^{2}}},
$$

where $P_{\min }$ is the required minimum power for tag operation, $M_{d}$ means the modulation depth, and $\lambda_{i}^{k}$ is the wavelength of $R_{i}$ in the $k$ th slot.

Accordingly, $r_{i}^{k}$ can be determined by

$$
r_{i}^{k}=\min \left(r_{i, \mathrm{th}}^{k}, r_{i, \max }^{k}\right) .
$$

In R2RCAM, the utility of an RFID system is evaluated by the interrogation region area of all the readers which is defined as $S_{\mathrm{tt}}$. To maximize $S_{\mathrm{tt}}$, R2RCAM is formulated as

$$
\begin{aligned}
\operatorname{maximize} & S_{\mathrm{tt}}=\sum_{k=1}^{N_{S}} \sum_{i=1}^{N_{\mathrm{rd}}} \pi\left(r_{i}^{k}\right)^{2} \\
\text { s.t. } & \mathrm{CH}_{i}^{k} \in\left[1, N_{F}\right], \\
& \forall i \in\left[1, N_{\mathrm{rd}}\right], k \in\left[1, N_{S}\right] .
\end{aligned}
$$

2.3. Artificial Immune Network (aiNet). To solve theoretical or engineering problems which can be generalized as optimization problems, for example, R2RCAM, intelligent algorithms are generally employed. aiNet is a main member in artificial immune systems, which is enlightened by biological immune systems to solve real-world problems [11].

Take the canonical opt-aiNet [9] as an example to introduce the structure of aiNet. The opt-aiNet uses real number encoding and has five operators, that is, cloning, mutation, selection, suppression, and recruitment. In the cloning phase, opt-aiNet uses the uniform cloning operator, where a fixnumber cloning is conducted for each antibody individual, and all the clones except the parent one experience the Gaussian mutation following

$$
\mathrm{Ab}^{\prime}=\mathrm{Ab}+\frac{G}{\beta} \cdot \exp \left(-\mathrm{Aff}^{*}(\mathrm{Ab})\right)
$$

Then, only one antibody, whose affinity is the highest among offsprings, is selected to enter the next generation. In (6), G is a random variable following Gaussian distribution whose mean is zero and standard deviation is $1, \beta$ is the control coefficient, $\mathrm{Ab}^{\prime}$ is the mutated antibody of $\mathrm{Ab}$, and $\mathrm{Aff}^{*}$ is the function of normalized affinity in the interval $[0,1]$. If the average affinity looks stable, the suppression operator will be triggered. For any two antibodies, if their distance is smaller than a suppression threshold $\delta_{S}$, the one with lower affinity will be removed. Then, a number of antibodies are randomly generated and merged into the antibody population to keep the population diversity. Repeat the above process until it is terminated.

\section{The Proposed R2RCAM-IRO Optimized by RA-IRO-aiNet}

\subsection{R2RCAM-IRO}

3.1.1. Modeling of R2RCAM-IRO. To solve theoretical optimization problem, let us assume that $N_{\text {tg }}$ tags are distributed uniformly in the deployment region and the maximum transmitting power of readers is $P_{\max }$. And the other assumptions are the same as those of R2RCAM.

Taking into account the identification capacity of a reader in a slot, $r_{i}^{k}$ must satisfy

$$
N_{\operatorname{tg}} \frac{\pi\left(r_{i}^{k}\right)^{2}}{S_{0}} \leq N_{\text {tg,max }}^{i}
$$

where $N_{\mathrm{tg}, \max }^{i}$ is the maximum number of tags that $R_{i}$ can identify in one slot. As a result, we can get

$$
r_{i}^{k} \leq \sqrt{\frac{S_{0} N_{\mathrm{tg}, \max }^{i}}{\pi N_{\mathrm{tg}}}} .
$$

According to (3) and (8), $r_{i}^{k}$ is determined by

$$
r_{i}^{k}=\min \left(r_{i, \mathrm{th}}^{k}, r_{i, \max }^{k}, \sqrt{\frac{S_{0} N_{\mathrm{tg}, \max }^{i}}{\pi N_{\mathrm{tg}}}}\right) .
$$

Tag identification rate (TIR) is a measure of the RFID system performance. TIR is defined as the ratio of the number of identifiable tags to the total number of tags. Because of the assumption that the tags are distributed uniformly, TIR is 
equal to the ratio of the total interrogation area to the reader deployment region area. That is,

$$
\operatorname{TIR}=\frac{S_{\mathrm{tt}}}{\mathrm{S}_{0}} .
$$

In the canonical R2RCAM, $S_{\mathrm{tt}}$ is calculated easily by (5). However, there may be an overlap between the interrogation regions of some readers. Thus, the canonical R2RCAM does not meet the practice requirement and decreases TIR of the RFID system. Let $\mathrm{Rg}_{i}$ represent the interrogation region of the reader $R_{i}$. And in the proposed R2RCAM-IRO, the total interrogation region $\mathrm{Rg}$ follows

$$
\operatorname{Rg}=\bigcup_{i=1}^{N_{\mathrm{rd}}} \operatorname{Rg}_{i}=\bigcup_{i=1}^{N_{\mathrm{rd}}} \bigcup_{k=1}^{N_{S}} \operatorname{Rg}_{i}^{k}
$$

where $\mathrm{Rg}_{i}^{k}$ is the interrogation region of $R_{i}$ in the $k$ th slot.

Consequently, R2RCAM-IRO can be formulated as

$$
\begin{array}{cl}
\text { maximize } & \operatorname{TIR}=\frac{S_{\mathrm{tt}}}{S_{0}} \\
\text { s.t. } & \mathrm{CH}_{i}^{k} \in\left[1, N_{F}\right], \\
& P_{i}^{k} \in\left[0, P_{\max }\right] \\
& \forall i \in\left[1, N_{\mathrm{rd}}\right], k \in\left[1, N_{S}\right] .
\end{array}
$$

3.1.2. Analysis of R2RCAM-IRO. Assume that when the maximum TIR is obtained, a reader $R_{i}$ works in $k_{i, 1}$ th, $k_{i, 2}$ th, $\ldots, k_{i, p}$ th slot $\left(2 \leq p \leq N_{S}\right.$ and $\left.k_{i, p_{1}} \neq k_{i, p_{2}}, \forall p_{1} \neq p_{2}\right)$ and the interrogation radii are $r_{i}^{k_{i, 1}}, r_{i}^{k_{i, 2}}, \ldots, r_{i}^{k_{i, p}}$, respectively. Without loss of generality, it is assumed that $r_{i}^{k_{i, 1}} \geq r_{i}^{k_{i, 2}} \geq$ $\cdots \geq r_{i}^{k_{i, p}}$. As a result,

$$
\operatorname{Rg}_{i}=\bigcup_{m=1}^{p} \operatorname{Rg}_{i}^{k_{i, m}}=\operatorname{Rg}_{i}^{k_{i, 1}}
$$

This indicates that if $R_{i}$ just works in $k_{1}$ th slot, its interrogation area will not change. Assume that another reader $R_{j}$ $(j \neq i)$ works in $k_{j, 1}$ th, $k_{j, 2}$ th, ..., $k_{j, q}$ th slot $\left(1 \leq q \leq N_{S}\right.$, $k_{j, q_{1}} \neq k_{j, q_{2}}, \forall q_{1} \neq q_{2}$, and $\left.\exists k_{u} \in\left\{k_{i, 1}, k_{i, 2}, \ldots, k_{i, p}\right\}\right)$ and its interrogation radius in $k_{u}$ th slot is denoted by $r_{j}^{k_{u}}$. If $R_{i}$ does not work in $k_{u}$ th slot, the theoretical interrogation radius of $R_{j}$ will increase because its total interference decreases. With the constraints of the maximum reader interrogation radius and the identification capacity, the interrogation radius $r_{j}^{k_{u}{ }^{*}}$ may be unchanged. Thus, $r_{j}^{k_{u}{ }^{*}} \geq r_{j}^{k_{u}}$ and $\operatorname{Rg}_{j}^{k_{u}{ }^{*}} \supseteq \operatorname{Rg}_{j}^{k_{u}}$ can be obtained. Thus, the total interrogation region $\operatorname{Rg}_{j}{ }^{*}$ of $R_{j}$ satisfies

$$
\begin{aligned}
\operatorname{Rg}_{j}^{*} & =\operatorname{Rg}_{j}^{k_{u}^{*}} \cup \bigcup_{m=1, m \neq k_{u}}^{q} \operatorname{Rg}_{j}^{k_{m}} \supseteq \operatorname{Rg}_{j}^{k_{u}} \cup \bigcup_{m=1, m \neq k_{u}}^{q} \operatorname{Rg}_{j}^{k_{m}} \\
& =\operatorname{Rg}_{j} .
\end{aligned}
$$

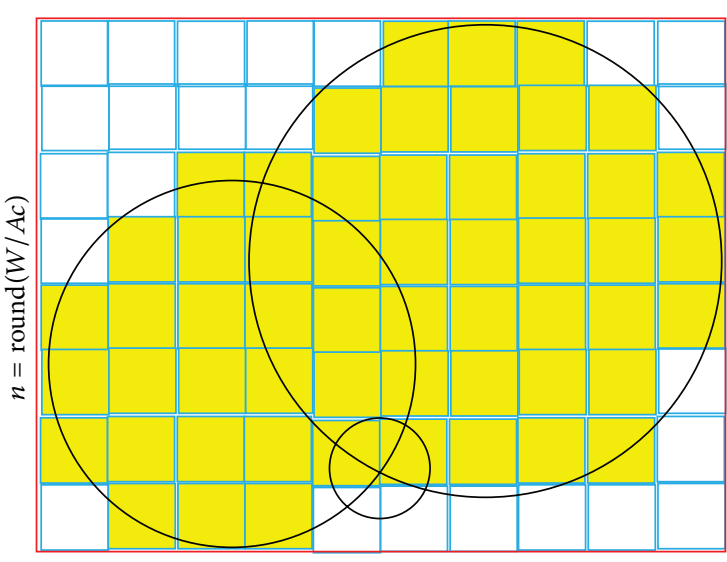

$m=\operatorname{round}(L / A c)$

FIGURE 3: Graphical representation of the grid method.

Thus, the total area of the interrogation regions of all the readers satisfies

$$
\mathrm{Rg}^{*}=\operatorname{Rg}_{j}{ }^{*} \cup \bigcup_{m=1, m \neq j}^{N_{\mathrm{rd}}} \operatorname{Rg}_{m} \supseteq \operatorname{Rg}_{j} \cup \bigcup_{m=1, m \neq j}^{N_{\mathrm{rd}}} \operatorname{Rg}_{m}=\mathrm{Rg} .
$$

Clearly, the total interrogation area of an RFID system when each reader just works in one slot is not less than that when some readers operate in two or more slots. Thus, only one slot can be allocated to each operating reader in R2RCAM-IRO.

3.1.3. Calculation Procedure of $S_{t t}$. Due to the interrogation region overlap, $S_{\mathrm{tt}}$ cannot be calculated directly. Thus, a grid method is employed to compute $S_{\mathrm{tt}}$. Taking Figure 3 as an example, the details of the grid method are depicted as follows.

Step 1. Determine the region of all the circles shown as the red rectangle with the length of $L$ and the width of $W$.

Step 2. Divide the region into $m \times n$ ( $m=$ round $(L / A c)$ and $n=$ round $(W / \mathrm{Ac})$ ) small rectangles with the area of $S_{\mathrm{sr}}$, where Ac determines the accuracy.

Step 3. Mark those small rectangles whose centers lie in any one of the circles as shown in yellow.

Step 4. Count the number $N_{\mathrm{mr}}$ of the marked small rectangles and use the total area of these rectangles as the result of $S_{\mathrm{tt}}$; that is, $S_{\mathrm{tt}}=S_{\mathrm{sr}} N_{\mathrm{mr}}$.

If the parameter Ac is large, the accuracy will be impaired. However, smaller Ac will result in a significant increase of the computational complexity. So, a preprocessing procedure is necessary to decrease the complexity by reducing the number of the circles and the area of the rectangular region. There are four situations for a single circle, where $(S 1)$ it lies in some circle; (S2) it is independent and does not intersect with any other circles; (S3) it intersects with only one circle; or (S4) it intersects with two or more circles. For any circle, if it is in $S 1$, it can be removed without having an impact on the result. 

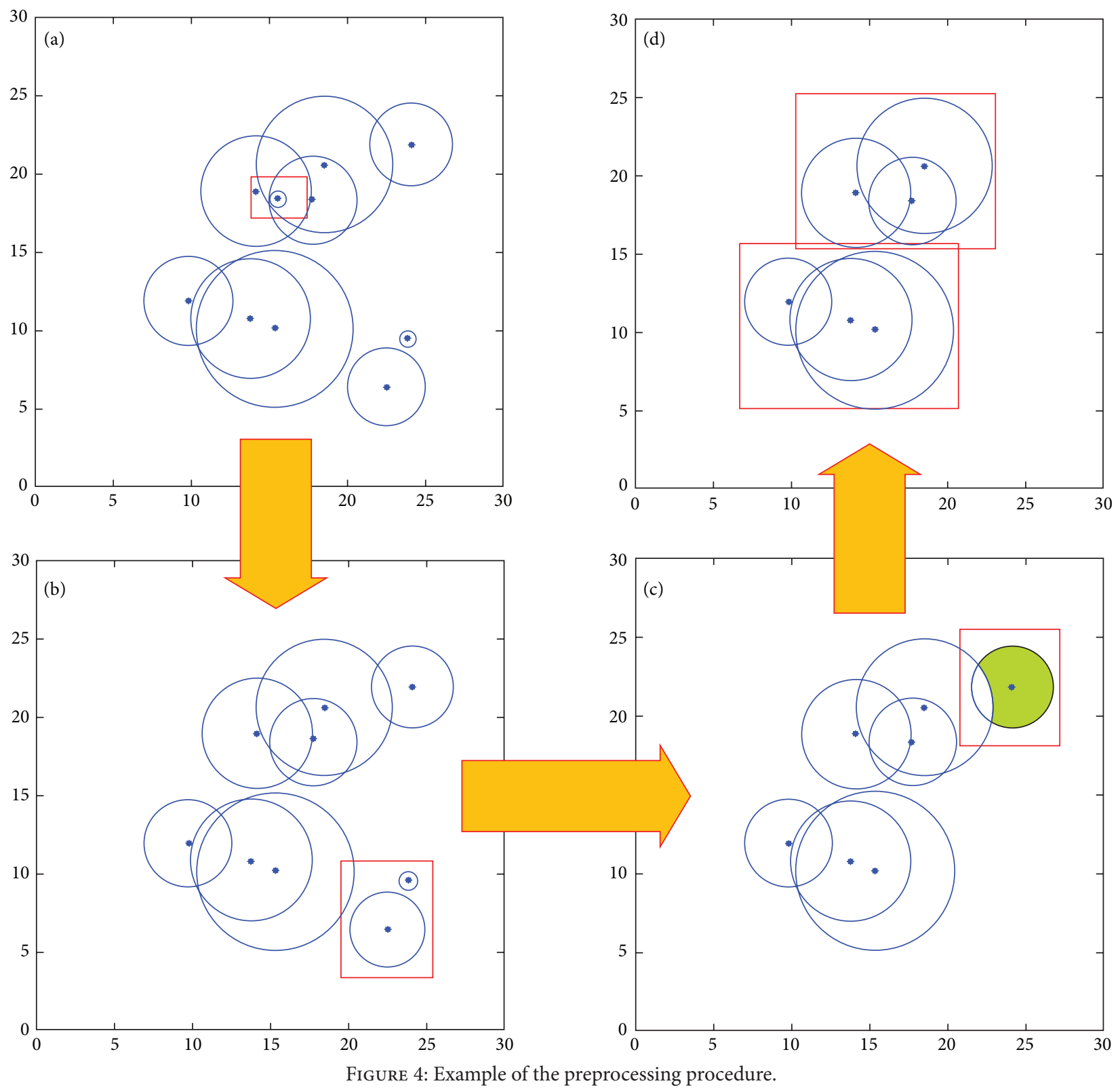

If it is in $S 2$, its area can be calculated easily. If it is in $S 3$, its area without the overlap is added to the result. And then, this circle can be removed. It is assumed that a circle $C_{i}$ with the centric point $O_{i}$ intersects with the other circle $C_{j}$ with the centric point $O_{j}$ at point $A$ and point $B$. And then the area $S_{\mathrm{ol}}$ of the overlap can be obtained by

$$
\begin{aligned}
S_{\mathrm{ol}} & =t_{i} r_{i}^{2}+t_{j} r_{j}^{2}-r_{i} d_{i j} \sin \left(t_{i}\right), \\
\cos \left(t_{i}\right) & =\frac{\left(r_{i}^{2}+d_{i j}^{2}-r_{j}^{2}\right)}{\left(2 r_{i} d_{i j}\right)}, \\
\cos \left(t_{j}\right) & =\frac{\left(r_{j}^{2}+d_{i j}^{2}-r_{i}^{2}\right)}{\left(2 r_{j} d_{i j}\right)},
\end{aligned}
$$

where $t_{i}$ is $\angle A O_{i} O_{j}, t_{j}$ is $\angle A O_{j} O_{i}$, and $d_{i j}$ is the distance from the point $O_{i}$ to the point $O_{j}$. For $S 4$, the grid method is employed.

Taking Figure 4 as an example, the details of the preprocessing procedure are depicted as follows.

Step 1. Find the circles in some other circles, such as the one in the red solid box in Figure 4(a). And then remove it.

Step 2. Find the independent circles, such as the two in the red solid box in Figure 4(b). Calculate their area and add it to the result. And then remove them.

Step 3. Find the circles intersected with only one other circle, such as the one in the red solid box in Figure 4(c). Calculate the area without the overlap as shown in green by (16) and add it to the result. And then remove it. 
TABLE 2: Encoding format for the antibody individual.

\begin{tabular}{lcc}
\hline Slot segment & Channel segment & Power segment \\
\hline$\left(T_{i, 1}, T_{i, 2}, \ldots, T_{i, N_{\mathrm{rd}}}\right)$ & $\left(\mathrm{Ch}_{i, 1}, \mathrm{Ch}_{i, 2}, \ldots, \mathrm{Ch}_{i, N_{\mathrm{rd}}}\right)$ & $\left(P_{i, 1}, P_{i, 2}, \ldots, P_{i, N_{\mathrm{rd}}}\right)$ \\
\hline
\end{tabular}

Step 4. If new independent circles or ones intersected with another circle appear, repeat Steps 2 and 3 until any circle intersects with two or more other ones.

Step 5. Separate the rest of the circles into groups, where the intersected circles belong to the same group and circles in different groups do not intersect with each other, as shown in Figure 4(d). And the area of each group is calculated by the grid method.

3.2. RA-IRO-aiNet. By improving the main immune operators, artificial immune networks become efficient, adaptive, and suitable for the practical applications. To find the optimal scheme of time slots, channels, and power for an RFID reader network, this subsection designs RA-IRO-aiNet to solve R2RCAM-IRO. The technical details of the proposed algorithm are introduced in the following subsections.

3.2.1. Encoding Format. A hybrid encoding format is used in RA-IRO-aiNet uses. Table 2 presents the encoding format used by the $i$ th antibody. In Table 2, each antibody includes three segments of the slot, the channel, and the power, where $T_{i, j} \in\left[0, N_{S}\right]$ is an integer and denotes the time slot, $\mathrm{Ch}_{i, j} \in$ $\left[1, N_{F}\right]$ is an integer and denotes the channel, and $P_{i, j} \in$ $\left(0, P_{\max }\right]$ is a real number and denotes the power. Note that $T_{i, j}=0$ means that $R_{j}$ does not operate.

3.2.2. Affinity Function. To evaluate each antibody individual about the resource scheduling solution, an affinity function is designed according to R2RCAM-IRO presented in Section 2, which is presented as

$$
\operatorname{Aff}(\mathrm{Ab})=\frac{S_{\mathrm{tt}}}{S_{0}}
$$

3.2.3. Initialization. In the initialization, an antibody population with $N_{\mathrm{ab}}$ individuals is constructed randomly. And each antibody must be feasible, where $T_{i, j} \in\left\{0,1, \ldots, N_{S}\right\}, \mathrm{Ch}_{i, j} \in$ $\left\{1,2, \ldots, N_{\mathrm{Ch}}\right\}$, and $P_{i, j} \in\left(0, P_{\mathrm{max}}\right]$ for $i \in\left\{1,2, \ldots, N_{\mathrm{ab}}\right\}$ and $j \in\left\{1,2, \ldots, N_{\text {rd }}\right\}$.

3.2.4. Cloning Operator. The uniform cloning operator is used, where each antibody is reproduced for a constant multiplier of $N_{C}$. The uniform cloning is simple to implement and can remarkably depress the computational consumption. All the cloned offsprings except the parent antibodies will execute the following mutation operator in order to increase the population diversity.

3.2.5. Mutation Operator. Because the proposed RA-IROaiNet uses hybrid encoding, a differentiated mutation operator is employed, in which different segments (i.e., the slot, the channel, and the power) of each antibody experience different

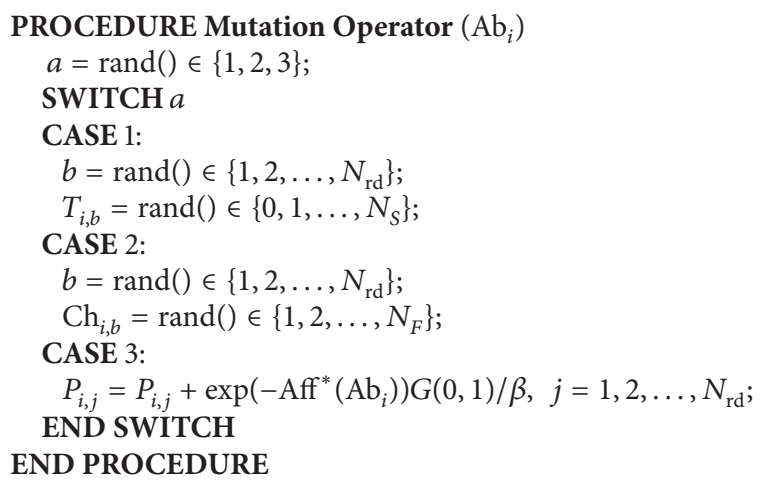

Pseudocode 1: Pseudocode of the mutation process.

mutation processes. In detail, the random uniform mutation operator is employed for the slot segment and the channel segment, where a randomly selected bit is changed randomly in its set of feasible solutions. And for the power segment, the used Gaussian mutation operator is given in (6). Note that only one randomly selected segment experiences the mutation for each offspring. The pseudocode of the mutation process is shown in Pseudocode 1.

3.2.6. Suppression Operator and Recruitment. The similarity between any two antibodies is evaluated by the measure of hybrid distance $\left(D_{\mathrm{Hyb}}\right)$. It is obtained by the sum of the normalized distance of each segment. The corresponding formula is given in (18)

$$
\begin{aligned}
D_{\mathrm{Hyb}}\left(\mathrm{Ab}_{i}, \mathrm{Ab}_{j}\right)= & \frac{D_{\mathrm{Ham}, T}\left(\mathrm{Ab}_{i}, \mathrm{Ab}_{j}\right)}{N_{\mathrm{rd}}} \\
& +\frac{D_{\mathrm{Ham}, \mathrm{Ch}}\left(\mathrm{Ab}_{i}, \mathrm{Ab}_{j}\right)}{N_{\mathrm{rd}}} \\
& +\frac{D_{\mathrm{Euc}, P}\left(\mathrm{Ab}_{i}, \mathrm{Ab}_{j}\right)}{D_{\max }}
\end{aligned}
$$

where $D_{\mathrm{Ham}, T}(\cdot, \cdot)$ and $D_{\mathrm{Ham}, \mathrm{Ch}}(\cdot, \cdot)$ are the Hamming distance of the slot segment and the channel segment, respectively, $D_{\text {Euc, } P}(\cdot, \cdot)$ is the Euclidean distance of the power segment, and $D_{\max }$ is the maximum Euclidean distance which equals $P_{\max } \sqrt{N_{\text {rd }}}$. The higher the hybrid distance is, the better diversity the antibody population has. Thus, in order to keep the diversity of the antibody population, once the hybrid distance of two antibodies is smaller than a threshold $\delta_{S}$, the antibody with lower affinity will be suppressed. The threshold is determined by such defined similarity following

$$
\begin{aligned}
\delta_{S} & =\min \left\{D_{\mathrm{Hyb}}\left(\mathrm{Ab}_{i}, \mathrm{Ab}_{j}\right), i \neq j\right\} \\
& +\xi\left(\max \left\{D_{\mathrm{Hyb}}\left(\mathrm{Ab}_{i}, \mathrm{Ab}_{j}\right)\right\}\right. \\
& \left.-\min \left\{D_{\mathrm{Hyb}}\left(\mathrm{Ab}_{i}, \mathrm{Ab}_{j}\right)\right\}\right) .
\end{aligned}
$$

The desired goal is to adjust the threshold dynamically and to remove those $\xi(0<\xi<1)$ of similar antibodies. After 


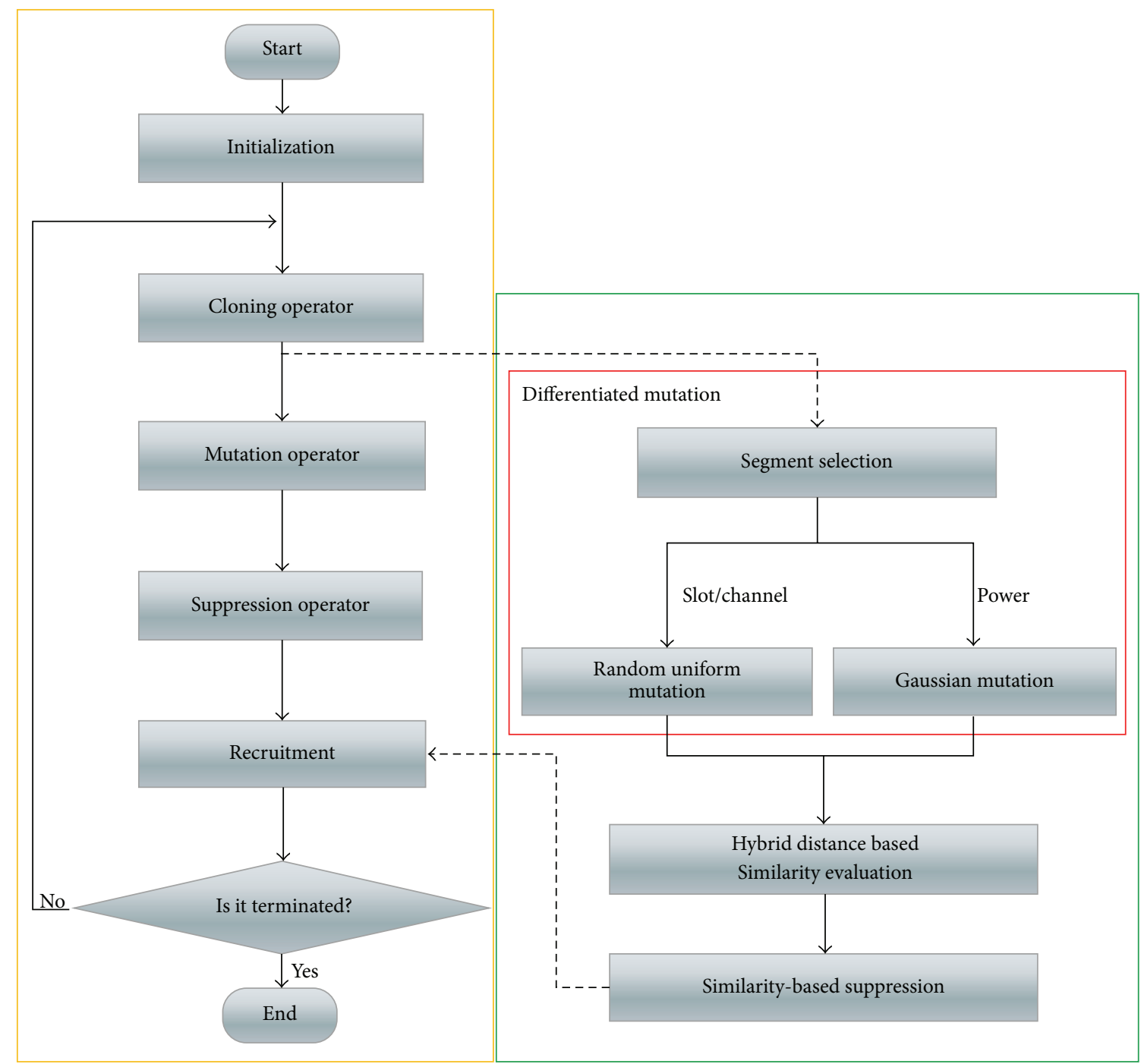

The left orange box: the canonical opt-aiNet

The right green box: the proposed RA-IRO-aiNet

FIGURE 5: Flowchart of the proposed RA-IRO-aiNet.

the suppression operator, some new antibodies are randomly produced to keep the antibody population at the number of $N_{\mathrm{ab}}$.

The flowchart of our proposed RA-IRO-aiNet is shown in Figure 5. The left orange box shows the flowchart of opt-aiNet, while the right green box highlights the revised immune operators of RA-IRO-aiNet. In RA-IRO-aiNet, each antibody experiences the uniform cloning operator, the differentiated mutation operator, the hybrid similarity evaluation, the similarity-based suppression operator, and the recruitment in each generation. Repeat the above process until it is terminated.

\section{Simulations and Results}

4.1. Environment for Simulation. In the numerical simulations, suppose that readers are randomly deployed in a square area of $100 \times 100 \mathrm{~m}^{2}$ and in the multiple-reader scenarios, where readers and tags transmit in the same channels following EPCglobal C1G2 [10]. All parameters in R2RCAM-IRO are listed in Table 3.

4.2. Simulation Results and Discussion. The effectiveness of the proposed R2RCAM-IRO optimized by the designed RAIRO-aiNet is discussed in this subsection. And furthermore, the efficiency of the designed RA-IRO-aiNet is examined by comparison with RA-GA [5], PSO [4], and opt-aiNet [9] in the total coverage area of the readers.

For all algorithms in the simulations, the population size is set to 50 , the maximum number of generations is set to 1000, and all the numerical simulations are repeated for 50 runs. In the proposed RA-IRO-aiNet, the encoding format has been introduced in Section 3.2.1, the cloned multiplier $N_{C}$ is 10 , the control parameter of mutation $\beta$ is 100 , and 
TABLE 3: Parameters in R2RCAM-IRO.

\begin{tabular}{lc}
\hline Parameter & Value \\
\hline Operating frequency & $902-928 \mathrm{MHz}$ \\
Bandwidth & $500 \mathrm{KHz}$ \\
Normalized spectrum power, $\alpha_{b \omega}$ & 0.86 \\
Reader antenna gain, $G_{T}, G_{R}$ & $6 \mathrm{dBi}$ \\
Reflection coefficient of effective power, $E_{\mathrm{tg}}$ & 0.1 \\
Required minimum power for tag operation, $P_{\text {min }}$ & $-15 \mathrm{dBm}$ \\
Reference path loss, $\mathrm{PL}_{0}$ & $-31.6 \mathrm{~dB}$ \\
Minimum SINR, SINR & $11.6 \mathrm{~dB}$ \\
Exponent of the path loss, $\gamma$ & 2.5 \\
Noise power, $N S$ & $-90 \mathrm{dBm}$ \\
Modulation depth, $M_{d}$ & 0.1 \\
Number of the tags, $N_{\text {tg }}$ & 100000 \\
Maximum identifiable tag number of a reader in a & 4000 \\
slot, $N_{\text {tg,max }}$ & 0.01 \\
Accuracy, Ac & \\
Spectrum mask, $\beta_{m}(\Delta \mathrm{CH})$ & $0 \mathrm{~dB}$ \\
$\Delta \mathrm{CH}=0$ & $-20 \mathrm{~dB}$ \\
$\Delta \mathrm{CH}=1$ & $-50 \mathrm{~dB}$ \\
$\Delta \mathrm{CH}=2$ & $-60 \mathrm{~dB}$ \\
$\Delta \mathrm{CH}=3$ & $-65 \mathrm{~dB}$ \\
$\Delta \mathrm{CH}>3$ &
\end{tabular}

the suppression parameter $\xi$ is $10 \%$. RA-GA uses hybrid encoding; in other words, the integer encoding is used in both the slot segment and the channel segment, and the 6bit binary encoding for $P_{i, j}$ is used in the power segment. PSO uses real number encoding. And, opt-aiNet also uses real number encoding and has the same cloned copies and control parameters of mutation to the proposed RA-IROaiNet. Other parameters of RA-GA, opt-aiNet, and PSO used in the simulations are referred to in $[4,5]$ and [9], respectively.

4.2.1. Effectiveness of the Proposed R2RCAM-IRO. Figure 6 shows TIRs from two different models: the proposed R2RCAM-IRO and R2RCAM. Note that the overlap is considered in R2RCAM-IRO but not in R2RCAM. As shown in Figure 6, TIRs grow nonlinearly as the number of readers increases and is less than 24 . When more readers are added, R2RCP become more serious, and thus the solution dimension increases more. It heavily degrades the convergence performance of RA-IRO-aiNet. Consequently, TIRs begin to decrease. These two models have similar TIR with less than 10 readers, while TIR of the proposed R2RCAM-IRO is much better than that of R2RCAM. For example, with 20 readers, the proposed R2RCAM-IRO harvests an improvement of $11.9 \%$.

Assume that 10 channels are available for 20 readers, and the maximum power is $1 \mathrm{~W}$. In this case, Figure 7 shows the effect of the number of available time slots on TIR. Seen from Figure 7, when the slot number increases from 3 to 15, TIR increases nonlinearly and is gradually approximate to $38 \%$

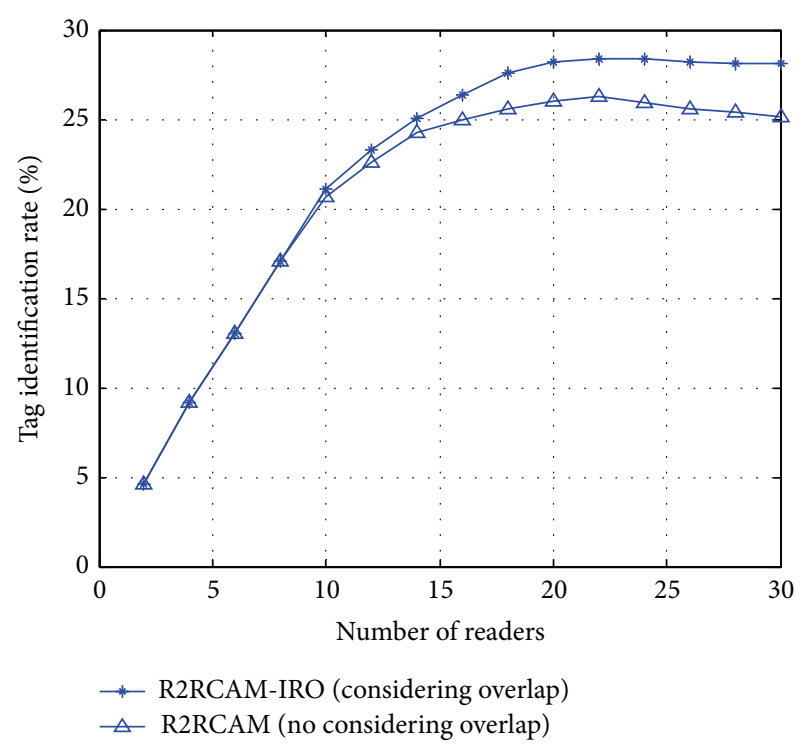

FIgURE 6: Tag identification rate.

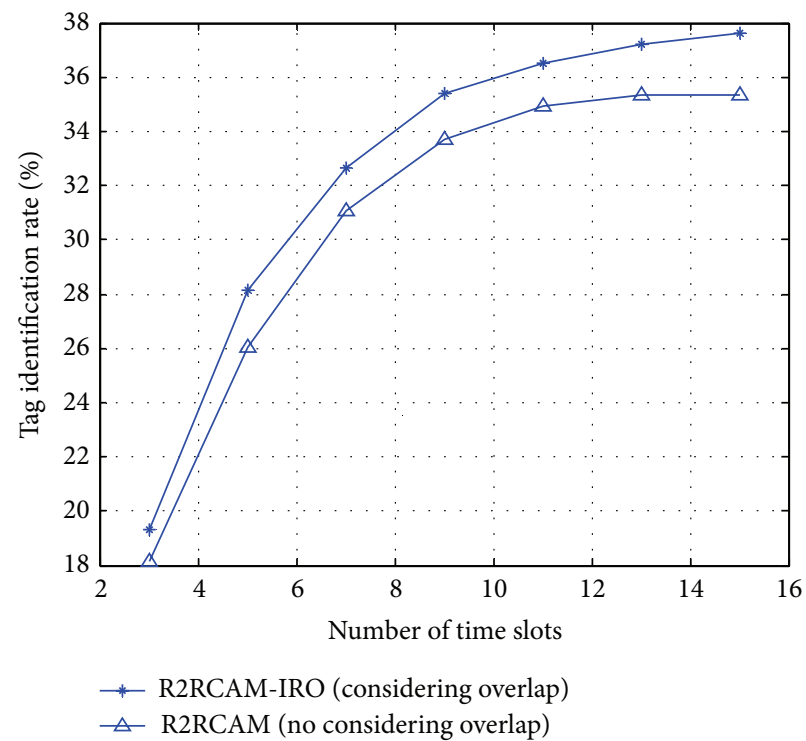

FigURE 7: Effect of the number of available time slots.

optimized by the proposed R2RCAM-IRO, while TIR is lower than $36 \%$, if R2RCAM is used.

Assume that 5 time slots are available for 20 readers, and the maximum power is $1 \mathrm{~W}$. In this case, Figure 8 presents the effect of the number of available channels. In Figure 8, TIRs of the two models are similar when the number available channels is less than 6 , while the proposed R2RCAM-IRO shows its superiority obviously with more available channels. In particular, with 20 channels, TIR of the proposed R2RCAM-IRO can reach $33.6 \%$, which improves R2RCAM by $16.7 \%$.

Assume that 5 time slots and 10 channels are available for 20 readers. In this case, Figure 9 describes the effect of the power. As shown in Figure 9, TIR increases when the power becomes greater and is approaching $31 \%$ for the proposed 


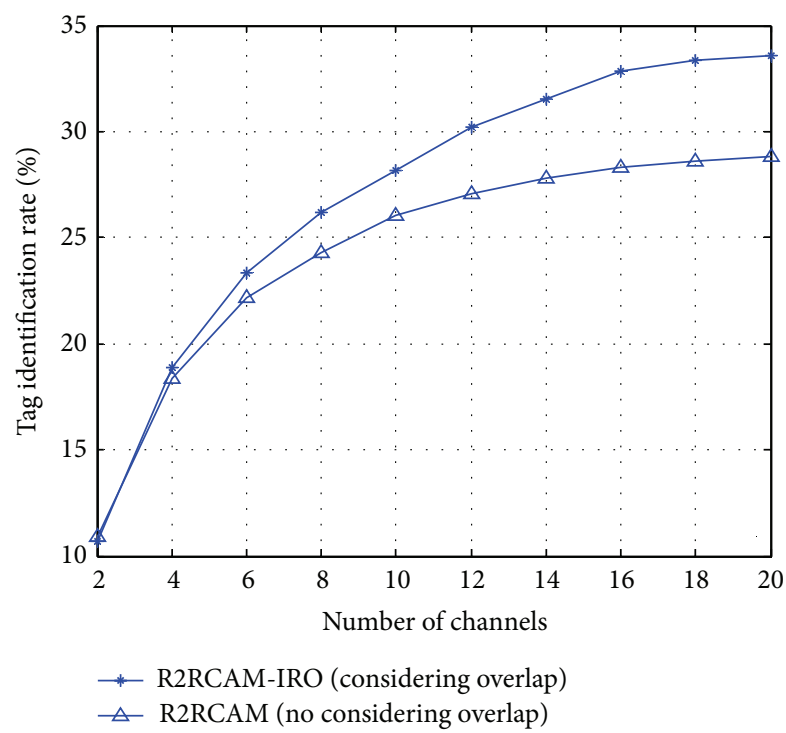

FIGURE 8: Effect of the number of available channels.

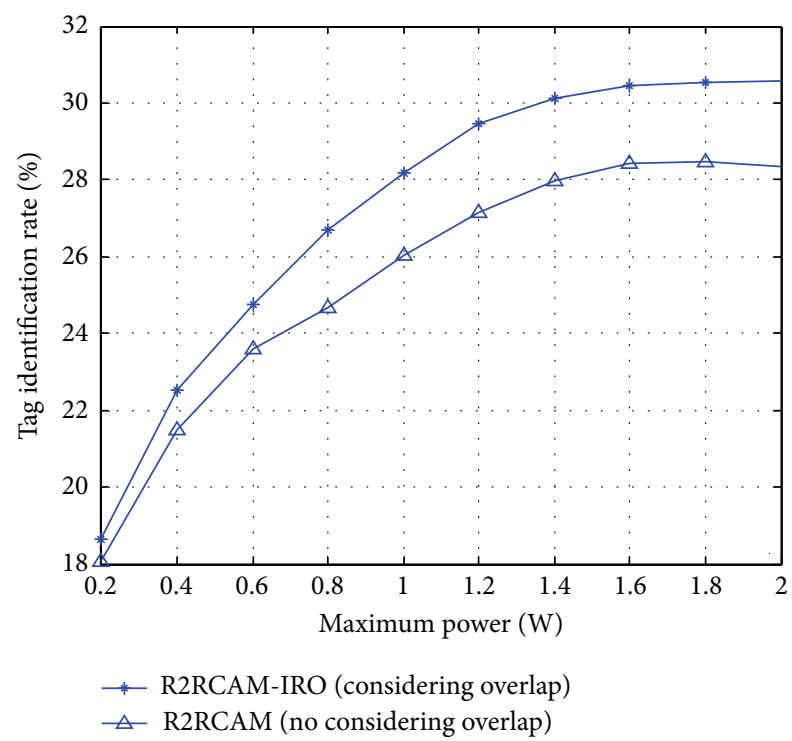

FIGURE 9: Effect of the power.

R2RCAM-IRO, while it is approaching 29\% for R2RCAM. The proposed R2RCAM-IRO improves TIR by 7.9\% with the maximum power of $2 \mathrm{~W}$ compared with R2RCAM.

The results from Figures 6-9 illustrate that R2RCAMIRO is effective and outperforms R2RCAM, where the overlap is ignored in the tag identification rate with limited resources.

4.2.2. Comparison in the Total Coverage Area of the Readers. Assume that 5 time slots and 10 channels are available for 20 readers, and the maximum power is $1 \mathrm{~W}$. In this case, Figure 10 shows the total coverage area of readers based on the proposed model optimized by RA-GA, opt-aiNet, PSO, and the proposed RA-IRO-aiNet.

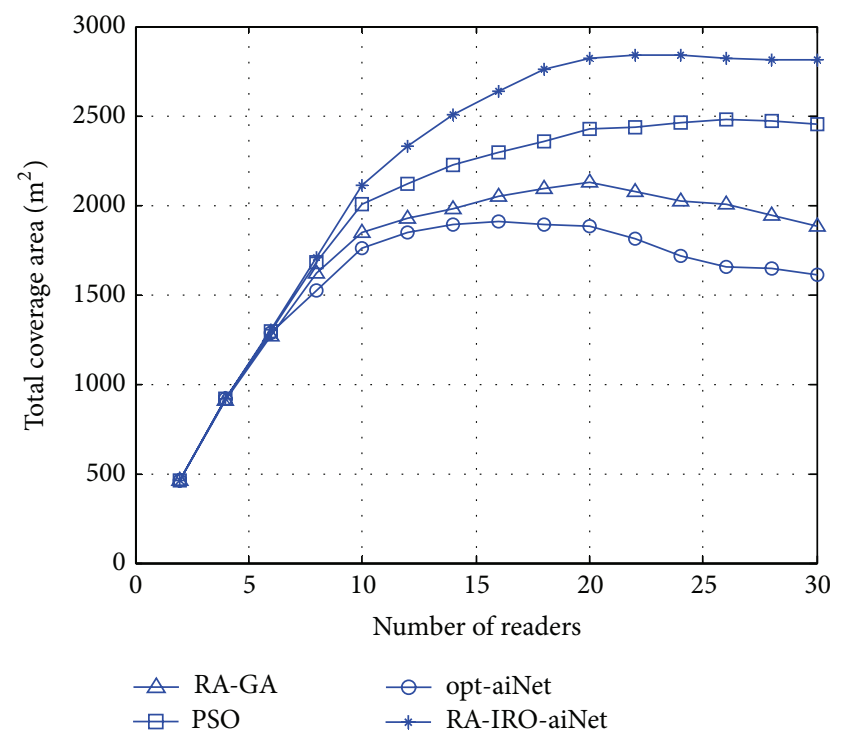

Figure 10: Total coverage area.

As shown in Figure 10, these four algorithms perform similarly to some extent in the optimal total coverage area with less than 6 readers. This is because the dimension of the solution is so low that each algorithm can capture the global optima and some desired solution. However, as more readers are added, the dimension of the solution becomes higher, which degrades the convergence performance. And the total coverage area optimized by RA-IRO-aiNet is much better than those obtained by the other algorithms. In particular, with 30 readers, the proposed algorithm has an improvement of $14.69 \%, 49.15 \%$, and $74.43 \%$ compared with RA-GA, optaiNet, and PSO, respectively.

Figure 11 gives the graphical representations of interrogation regions with 30 readers for the best solutions optimized by RA-GA, opt-aiNet, PSO, and the proposed RA-IRO-aiNet, respectively.

As shown in Figure 11, it is clear that the readers, where the proposed RA-IRO-aiNet is used, have much larger interrogation region than those by the other three algorithms. The proposed RA-IRO-aiNet has 16 operating readers with the average radius of $3.9887 \mathrm{~m}$, while RA-GA has 15 operating readers with the average radius of $3.4899 \mathrm{~m}$, PSO has 14 operating readers with the average radius of $3.6659 \mathrm{~m}$, and opt-aiNet has 15 operating readers with the average radius of $3.0091 \mathrm{~m}$. This indicates that the proposed RA-IRO-aiNet has larger average radius with more operating readers and outperforms the other three algorithms in interrogation regions especially in dense reader scenarios.

\section{Conclusions}

In this paper, the problem of RFID reader-to-reader collision is studied. And an enhanced collision avoidance model (i.e., R2RCAM-IRO) related to time slots, channels, and power is formulated. In the proposed model, the overlap between the interrogation regions of readers and the tag identification 


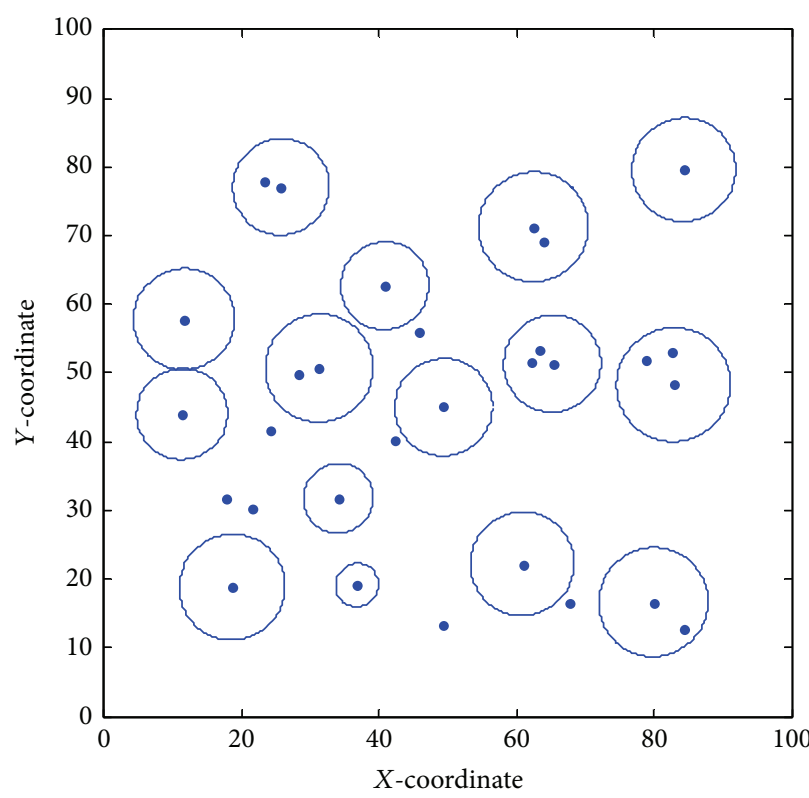

(a) RA-GA

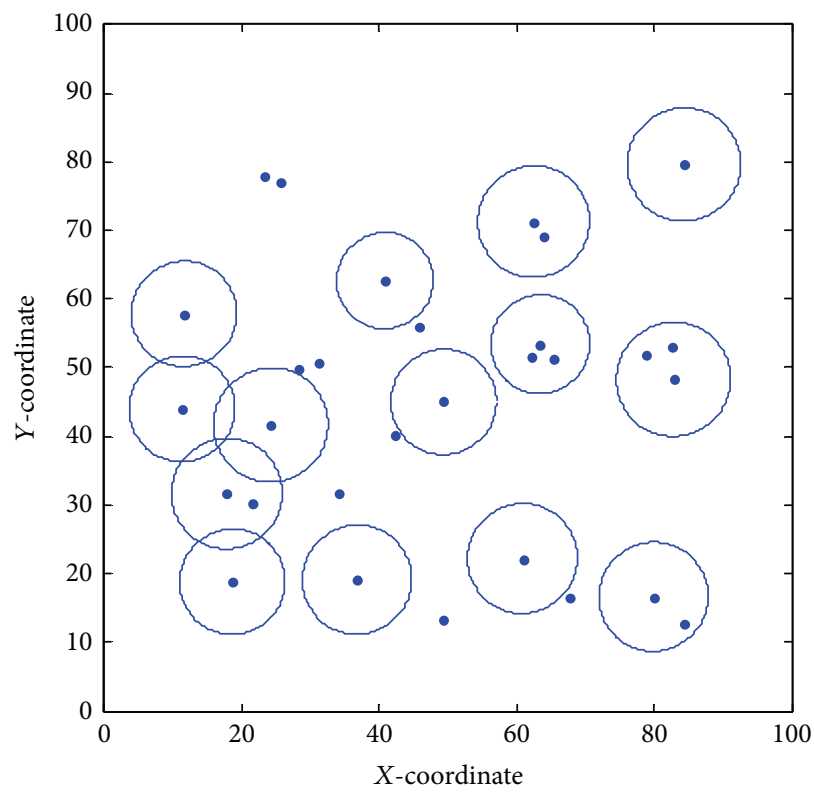

(c) $\mathrm{PSO}$

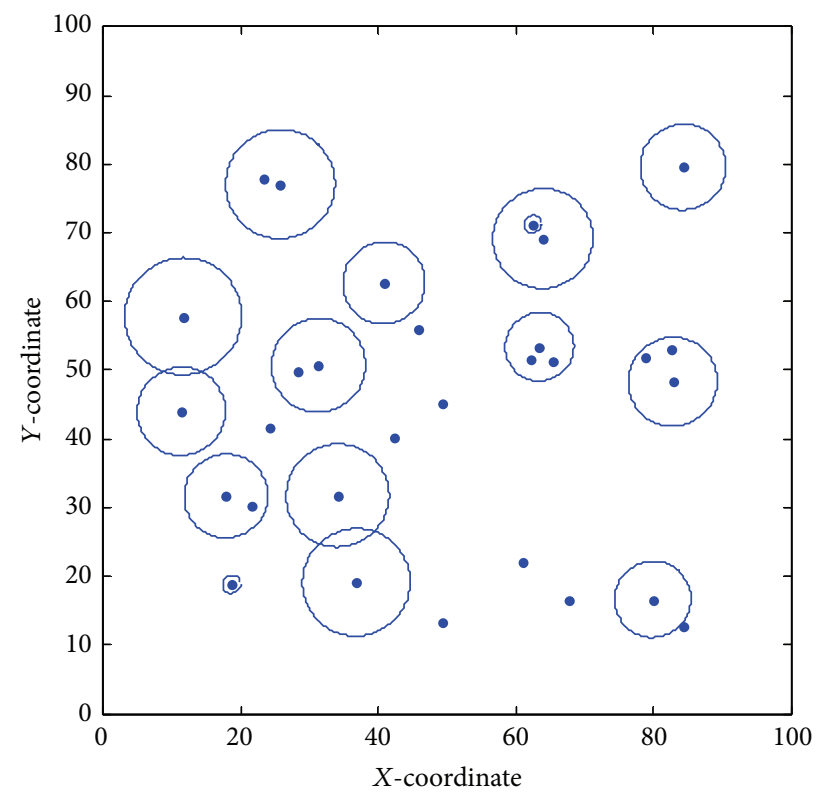

(b) opt-aiNet

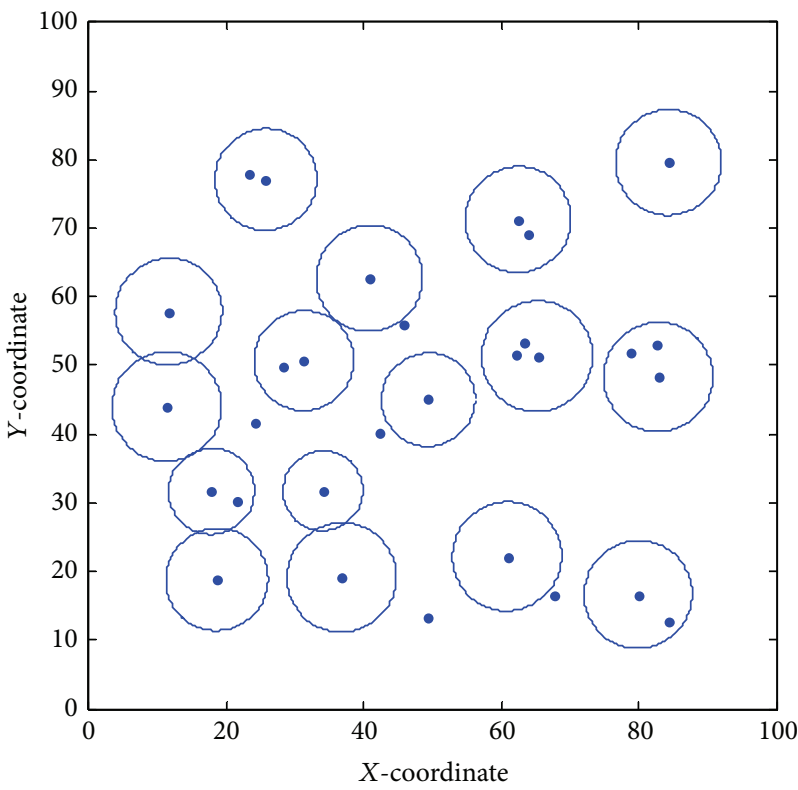

(d) The proposed RA-IRO-aiNet

FIGURE 11: Graphical representation of the readers' interrogation regions.

capacity of readers are considered. This makes R2RCAM-IRO suitable for the practice of RFID applications. In addition, a revised artificial immune network (i.e., RA-IRO-aiNet) is designed to optimize the proposed R2RCAM-IRO. In RAIRO-aiNet, the hybrid encoding, uniform cloning, differentiated mutation, hybrid distance based similarity evaluation, and similarity-based suppression are helpful to optimize the proposed R2RCAM-IRO. The simulation results show that the proposed R2RCAM-IRO is effective in tag identification rate with limited communication resources and the proposed RA-IRO-aiNet outperforms RA-GA, opt-aiNet, and PSO in the total coverage area of readers.

\section{Conflict of Interests}

The authors declare that there is no conflict of interests regarding the publication of this paper.

\section{Acknowledgments}

This work is partially supported by the National Nature Science Foundation of China under Grants nos. 61201087 and 61471122, the Guangdong Natural Science Foundation under Grant no. S201301001182, the Science and Technology Program of Huizhou under Grant nos. 2013B020015006 and 
2014B020004025, the Science and Technology Program of Zhaoqing under Grants nos. 2012G027 and 2013C003, and the Science and Technology Program of Guangzhou under Grant no. 2014J4100001.

\section{References}

[1] S. D. Park and H. Lee, "Self-recognition of vehicle position using UHF passive RFID tags," IEEE Transactions on Industrial Electronics, vol. 60, no. 1, pp. 226-234, 2013.

[2] G. M. Gaukler, "Item-level RFID in a retail supply chain with stock-out-based substitution," IEEE Transactions on Industrial Informatics, vol. 7, no. 2, pp. 362-370, 2011.

[3] C.-F. Lin and F. Y.-S. Lin, "A simulated annealing algorithm for RFID reader networks," in Proceedings of the IEEE Wireless Communications and Networking Conference (WCNC '07), pp. 1671-1674, IEEE, Kowloon, China, March 2007.

[4] T. Chen, Y. S. Yang, and B. Yang, "RFID dense reader network anti-collision PSO model and solving," in Proceedings of the $3 \mathrm{rd}$ International Conference on Intelligent Human-Machine Systems and Cybernetics (IHMSC '11), vol. 1, pp. 92-96, IEEE, Zhejiang, China, August 2011.

[5] H. Seo and C. Lee, "A new GA-based resource allocation scheme for a reader-to-reader interference problem in RFID systems," in Proceedings of the IEEE International Conference on Communications (ICC '10), pp. 1-5, Cape Town, South Africa, May 2010.

[6] Z. Li and C. He, "Optimal scheduling-based RFID readerto-reader collision avoidance method using artificial immune system," Applied Soft Computing Journal, vol. 13, no. 5, pp. 25572568, 2013.

[7] W. Alsalih, K. Ali, and H. Hassanein, "A power control technique for anti-collision schemes in RFID systems," Computer Networks, vol. 57, no. 9, pp. 1991-2003, 2013.

[8] W. Dong and X. Hao, "The optimization control of UHF reader transmit power," in Proceedings of the 2nd International Conference on Computer Science and Network Technology (ICCSNT '12), pp. 996-1000, IEEE, Changchun, China, December 2012.

[9] L. N. de Castro and J. Timmis, "An artificial immune network for multimodal function optimization," in Proceedings of the Congress on Evolutionary Computation (CEC '02), vol. 1, pp. 699-704, IEEE, Honolulu, Hawaii, USA, May 2002.

[10] EPCglobal, "EPC radio-frequency identity protocols class-1 generation-2 UHF RFID protocol for communications at $860 \mathrm{MHz}-960 \mathrm{MHz}$ version 1.0.9," EPCglobal Standard Specification, 2004.

[11] Z. Li, Y. Zhang, and H.-Z. Tan, "IA-AIS: an improved adaptive artificial immune system applied to complex optimization problems," Applied Soft Computing Journal, vol. 11, no. 8, pp. 46924700, 2011. 


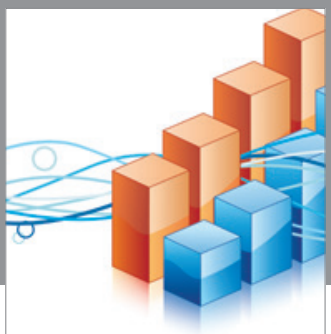

Advances in

Operations Research

vatem alat4

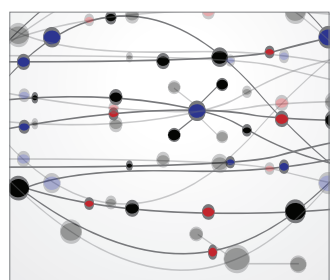

\section{The Scientific} World Journal
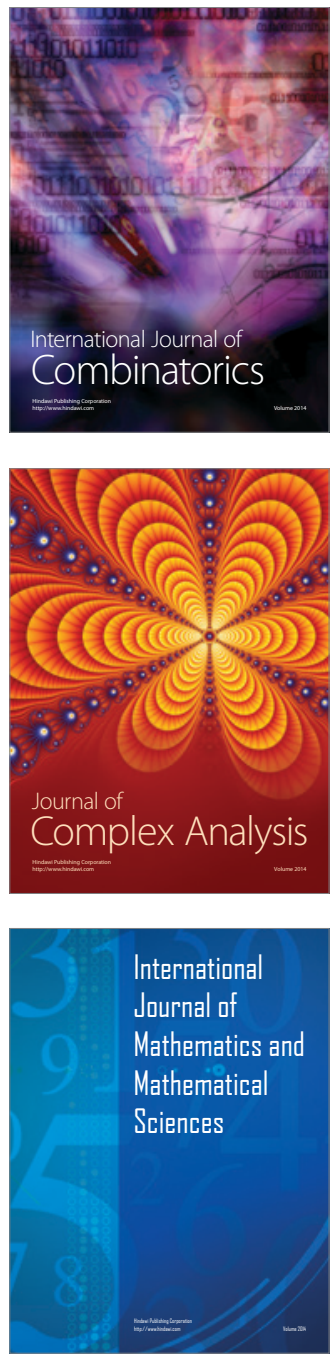
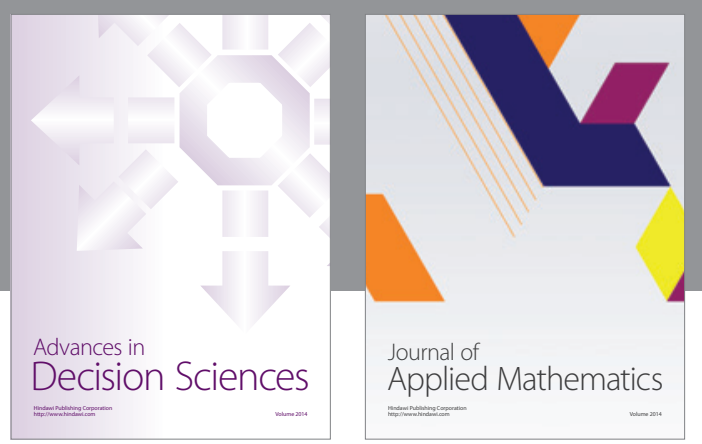

Algebra

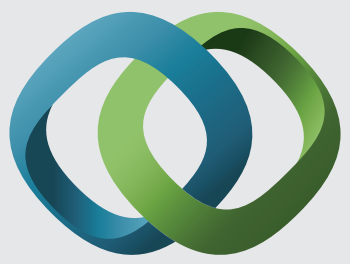

\section{Hindawi}

Submit your manuscripts at

http://www.hindawi.com
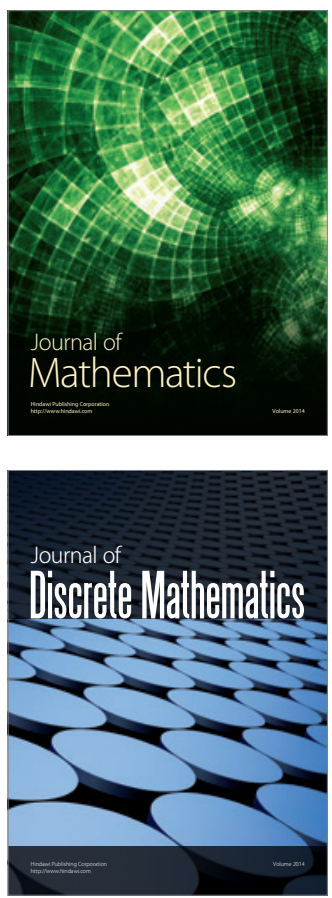

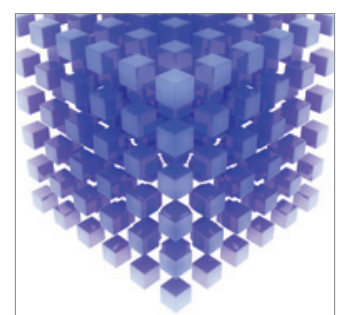

Mathematical Problems in Engineering
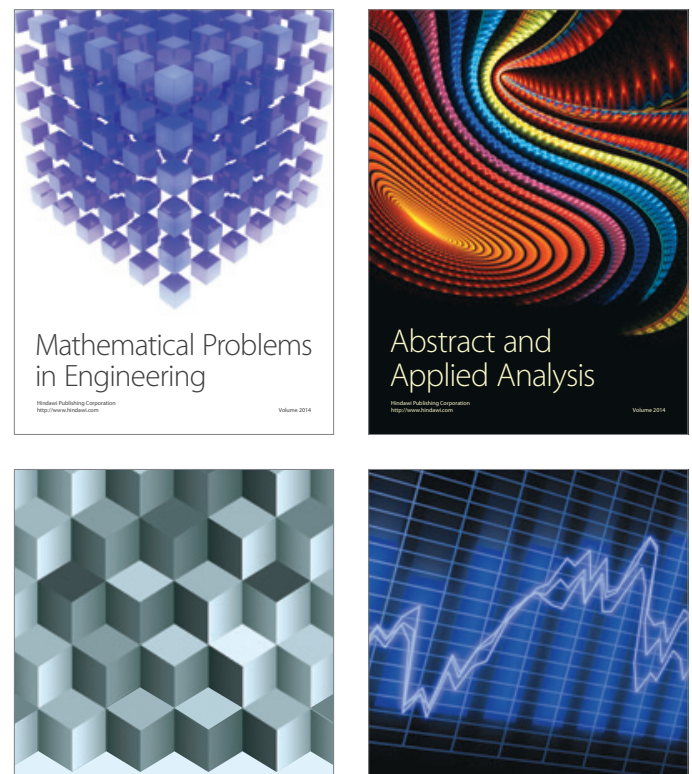

Journal of

Function Spaces

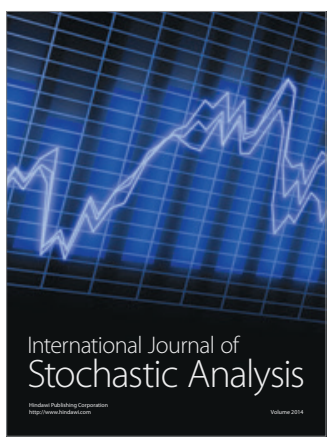

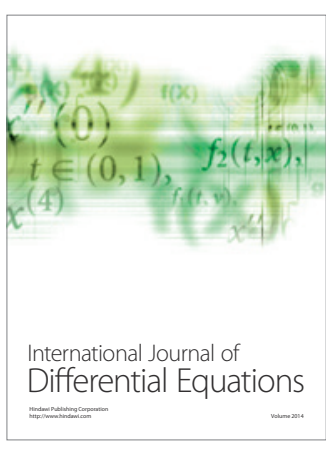
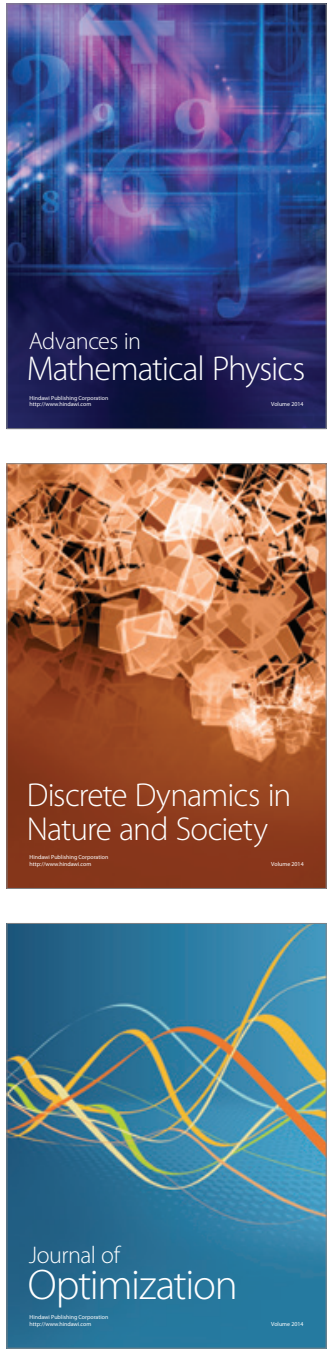